\title{
Relação entre Doença de Parkinson e Modulação Epigenética
}

\author{
Relationship Between Parkinson's Disease And Epigenetic \\ Modulation
}

\author{
Relación Entre La Enfermedad De Parkinson Y La \\ Modulación Epigenetica
}

\begin{abstract}
Mariana Marchioro ${ }^{1}$, Caroline Dani ${ }^{2}$, Viviane Elsner ${ }^{3}$, Cláudia Funchal $^{4}$
1.Biomédica, Discente do Programa de Pós-Graduação em Biociências e Reabilitação, Centro Universitário Metodista - IPA, Porto Alegre-RS, Brasil.

2.Biomédica, Mestre e Doutora em Biotecnologia, Docente do Programa de Pós-Graduação em Biociências e Reabilitação, Centro Universitário Metodista - IPA, Porto Alegre-RS, Brasil.

3.Fisioterapeuta, Mestre e Doutora em Ciências Biológicas: Fisiologia, Docente do Programa de PósGraduação em Biociências e Reabilitação, Centro Universitário Metodista - IPA, Porto Alegre-RS, Brasil. 4.Farmacêutica e Bioquímica, Mestre e Doutora em Ciências Biológicas: Bioquímica, Docente do Programa de Pós-Graduação em Biociências e Reabilitação, Centro Universitário Metodista - IPA, Porto Alegre-RS, Brasil.
\end{abstract}

\begin{abstract}
Resumo
Introdução. A Doença de Parkinson (DP) é um distúrbio neurodegenerativo com prevalência na população longeva. Apesar dos avanços científicos na busca da sua etiologia, os mecanismos celulares e moleculares desencadeadores da neurodegeneração não são bem compreendidos. Ainda que se tenha aprimorado os desfechos clínico-funcionais, os tratamentos disponíveis não previnem a progressão da doença. Sendo assim, há necessidade de estudos que investiguem mecanismos associados com a fisiopatologia e progressão da DP, bem como identifiquem biomarcadores para diagnóstico precoce e tratamentos mais eficazes. Neste contexto, a epigenética tem sido alvo de intensa investigação devido ao seu possível envolvimento em doenças neurodegenerativas, como na DP. Objetivo. Revisar na literatura científica os principais mecanismos epigenéticos envolvidos na DP. Método. Foi realizada uma revisão de literatura baseada em artigos científicos indexados nas bases de dados Medline e Lilacs no período de 2010 a 2018. Resultados. Os artigos relataram alterações nos mecanismos epigenéticos em modelos experimentais da DP bem como em pacientes. Conclusão. Deste modo, esperamos que este campo de estudo forneça importantes mecanismos moleculares que contribuam para a compreensão da patogênese da DP, assim como no desenvolvimento de biomarcadores de diagnóstico prematuro e abordagens terapêuticas mais efetivas para esta patologia.
\end{abstract}

Unitermos. Doença de Parkinson; epigenética; doenças neurodegenerativas; biomarcadores

\begin{abstract}
Introduction. Parkinson's disease (PD) is a neurodegenerative disorder prevalent in the elderly population. Despite scientific advances in the search for its etiology, the cellular and molecular mechanisms that trigger neurodegeneration are still not well understood. Although the clinical-functional outcomes have been improved, the available treatments do not prevent the progression of the disease. Therefore, there is a necessity for studies investigating mechanisms associated with the pathophysiology and progression of PD, as well as identifying biomarkers for early diagnosis and treatments that are more effective. In this context, epigenetics has been the subject of intense investigation due to its possible involvement in neurodegenerative diseases, as in PD. Objective. To review in the scientific literature the main epigenetic mechanisms involved in PD. Method. A literature review was carried out based on scientific articles indexed in Medline and Lilacs databases, from 2010 to 2018. Results. The articles reported alterations in epigenetic mechanisms in experimental models of PD as well as in patients. Conclusion. Thus, we hope that this field of study provides important molecular mechanisms that contribute to the understanding of the
\end{abstract}


pathogenesis of PD, as well as the development of biomarkers of premature diagnosis and more effective therapeutic approaches for this pathology.

Keywords. Parkinson's disease; epigenetics; neurodegenerative diseases; biomarkers

\section{Resumen}

Introducción. La enfermedad de Parkinson (EP) es un trastorno neurodegenerativo frecuente en la población de larga vida. A pesar de los avances científicos en la búsqueda de su etiología, los mecanismos celulares y moleculares que desencadenan la neurodegeneración no se conocen bien. Aunque los resultados clínico-funcionales han mejorado, los tratamientos disponibles no previenen la progresión de la enfermedad. Por lo tanto, existe la necesidad de estudios que investiguen los mecanismos asociados con la fisiopatología y la progresión de la EP, así como que identifiquen biomarcadores para el diagnóstico temprano y tratamientos más efectivos. En este contexto, la epigenética ha sido objeto de una intensa investigación debido a su posible participación en enfermedades neurodegenerativas, como la EP. Objetivo. Revisar en la literatura científica los principales mecanismos epigenéticos involucrados en la EP. Método. Realizamos una revisión de la literatura basada en artículos científicos indexados en las bases de datos Medline y Lilacs de 2010 a 2018. Resultados. Los artículos informaron cambios en los mecanismos epigenéticos en modelos experimentales de EP, así como en pacientes. Conclusión. Por lo tanto, esperamos que este campo de estudio proporcione mecanismos moleculares importantes que contribuyan a la comprensión de la patogénesis de la EP, así como al desarrollo de biomarcadores de diagnóstico temprano y enfoques terapéuticos más efectivos para esta patología.

Palabras clave. enfermedad de Parkinson; epigenética; enfermedades neurodegenerativas; biomarcadores

Trabalho realizado Centro Universitário Metodista - IPA, Porto Alegre-RS, Brasil.

\section{INTRODUÇÃO}

\section{A DP é a segunda desordem neurodegenerativa mais} frequente, afetando aproximadamente 1 a $2 \%$ da população com mais de 65 anos e atingindo uma prevalência de quase $4 \%$ nos idosos acima de 85 anos $^{1}$. Esta patologia é ocasionada pela degeneração dos neurônios dopaminérgicos na pars compacta da substância nigra, região responsável principalmente pelo controle da atividade motora. A DP é clinicamente caracterizada por bradicinesia, tremor de repouso, rigidez, anormalidades da 
marcha e da postura, além de outros sintomas não motores, como distúrbios do sono, disfunções autonômicas e comprometimentos cognitivos ${ }^{2}$.

Os deficits motores geralmente manifestam-se quando o paciente se encontra em um estágio avançado da neurodegeneração, sendo estimado uma perda de até $60 \%$ dos neurônios dopaminérgicos na substância nigra antes do diagnóstico clínico ${ }^{3}$. Agregados proteicos constituídos principalmente pela proteína alfa-sinucleína denominados corpos de Lewy, formam-se no citoplasma dos neurônios e, em combinação com a perda de neurônios dopaminérgicos na substância nigra, representam os achados fisiopatológicos da doença 4 .

Devido ao aumento da expectativa de vida, o número de indivíduos afetados pela DP aumentou consideravelmente, resultando em extensos desafios socioeconômicos. Diversas opções terapêuticas estão atualmente disponíveis para tratar a sintomatologia associada à DP. No entanto, ainda não há cura ou estratégia preventiva ${ }^{5}$. A maioria dos casos da doença são esporádicos, de origem idiopática, e acredita-se que resultam de interações complexas entre a suscetibilidade genética, toxinas ambientais, disfunções mitocondriais e outros processos celulares, mas ainda não bem compreendidos $^{6}$. Nesse contexto, evidências sugerem o envolvimento da modulação epigenética na fisiopatologia e progressão da DP7. 
O termo epigenética refere-se a modificações na expressão gênica que ocorrem independentemente de mudanças na sequência primária do DNA e são adquiridas ao longo da vida. Este processo pode ser decorrente de fatores ambientais, tais como estilo de vida como a dieta, prática de exercício físico e exposição a toxinas, resultando em mudanças fenotípicas ${ }^{8-11}$. Além de afetar a expressão gênica, as modificações epigenéticas desempenham também um papel vital no desenvolvimento, na regeneração e em doenças humanas ${ }^{12}$. No sistema nervoso central, muitas funções neurobiológicas e cognitivas como a neurogênese, o aprendizado e a plasticidade sináptica são reguladas por processos epigenéticos ${ }^{12-14}$. Estas modificações podem ser implementadas via metilação do DNA, modificações pós-traducionais de histonas ou pela regulação por RNAs não codificantes, principalmente os microRNAs ${ }^{12}$.

A metilação do DNA é mediada por uma família de enzimas chamadas DNA metiltransferases (DNMTs). Elas promovem a adição de um grupamento metil ao quinto carbono da base de DNA citocina, geralmente seguida por guanina (dinucleotídeo CpG). Esses dinucleotídeos não estão distribuídos de forma regular ao longo do genoma humano, porém, encontram-se concentrados em curtos trechos de DNA ricos em CpG, as chamadas "ilhas CpG"15. As sequências hipermetiladas desencadeiam mecanismos adicionais que resultam na condensação da cromatina, inibindo a transcrição da região promotora do gene e 
diminuindo a sua expressão, enquanto que as áreas hipometiladas a expressão gênica está ativa ${ }^{16}$.

A metilação do DNA é o mecanismo epigenético mais intensamente estudado, mas o papel que desempenha na patogênese da DP está apenas começando a ser explorado. A metilação do DNA se correlaciona com o envelhecimento dos tecidos e com o comprometimento cognitivo e influencia diretamente a expressão do gene SNCA e outros relacionados à $\mathrm{DP}^{17}$.

O remodelamento da cromatina, orquestrado pela modificação das histonas, é um processo dinâmico pelo qual a expressão gênica é regulada. As modificaçãos das histonas mais estudadas são a metilação e acetilação, sendo que estas podem alterar o estado conformacional da cromatina e assim, regular a acessibilidade do DNA para transcrição ${ }^{17}$. A acetilação das histonas é associada a genes transcricionalmente ativos. Esta modificação é mediada pelas enzimas histonas acetiltransferases (HATs), que catalisam a adição do grupamento acetil às lisinas presentes na cauda $\mathrm{N}$-terminal das histonas. Este processo acaba por enfraquecer as interações eletrostáticas do DNA com as histonas, levando ao relaxamento da cromatina, o que facilita a ação dos fatores de transcrição e ao aumento da expressão gênica. Enquanto que as histonas desacetilases (HDACs) medeiam o processo contrário, ou seja, removem o grupo acetil, sendo que sua ação resulta em uma maior associação do DNA com as histonas, 
tornando a cromatina mais condensada, favorecendo então, a repressão gênica ${ }^{18}$.

A metilação das histonas é catalisada pelas histonas metiltranferases (HMTs), enquanto a desmetilação ocorre por ação das histonas desmetilases (HDMs). A metilação das histonas pode ocasionar tanto o aumento da expressão gênica ou a diminuição. Isto irá depender de fatores como a histona e a lisina nas quais ocorrerá a adição do grupo metil e também o grau de metilação (monometilação, dimetilação ou trimetilação) ${ }^{19}$.

Deste modo, a participação de mecanismos epigenéticos vem sendo recentemente investigada com a finalidade de alcançar maior esclarecimento quanto à etiologia das doenças, podendo auxiliar no avanço de novos métodos diagnósticos e novas formas de tratamento. Modificações no controle da expressão gênica têm sido descritas em transtornos neurodegenerativos, as quais normalmente se alteram com o envelhecimento, portanto, poderiam contribuir para os processos deletérios descritos na $D P^{20,21}$. Sendo assim, o objetivo deste trabalho foi revisar na literatura científica os principais mecanismos epigenéticos envolvidos na DP.

\section{MÉTODO}

Este estudo é uma revisão descritiva, constituída por publicações relacionadas ao tema proposto, com significativa relevância, mediante pesquisa online, 
utilizando as seguintes bases de dados: Medline e Lilacs. Foram utilizados como critério para inclusão artigos escritos em português e inglês disponíveis na íntegra nas modalidades original ou revisão. Foram selecionados para compor esta pesquisa os artigos publicados desde o ano de 2010 até o ano 2018, como critério de exclusão os artigos que não estivessem na íntegra e que não se relacionassem com as palavras-chaves e seus respectivos termos em inglês. As palavras-chaves utilizadas para pesquisa foram: Doença de Parkinson, epigenética, doenças neurodegenerativas, encontradas de forma isolada ou combinadas.

Foram incluídos estudos que abordassem os mecanismos e modificações epigenéticas relacionados com o processo de envelhecimento, mais especificamente na DP. A consulta foi realizada sem restrições quanto ao delineamento do estudo, incluindo artigos originais (estudos clínicos e pré-clínicos), revisões de literatura e sistemáticas. Essa pesquisa foi realizada em 3 etapas: inicialmente foram analisados os títulos dos artigos encontrados, na segunda fase o resumo dos artigos foi analisado e na etapa final os trabalhos selecionados na etapa anterior foram lidos na íntegra. Outras fontes também foram utilizadas como livros. 


\section{RESULTADOS e DISCUSSÃO}

Foram encontrados 280 artigos com os termos utilizados nas bases de dados, sendo excluídos 263. A seleção dos artigos considerou aqueles de maior relevância de acordo com a abrangência ao tema proposto. No total foram selecionados 17 artigos, dentre eles, 12 estudos clínicos, um estudo de revisão sistemática e quatro estudos de revisão da literatura.

Acredita-se que a desregulação epigenética desempenhe um papel importante e pouco compreendido na etiopatogênese de vários distúrbios neurodegenerativos, incluindo a DP. Alterações na expressão gênica e nos níveis proteicos são a principal consequência dos mecanismos epigenéticos ${ }^{22}$.

A identificação de genes diferencialmente metilados e o processo no qual a metilação é mantida ou perdida tornou-se recentemente uma área importante de interesse na pesquisa de doenças neurodegenerativas. O envelhecimento está associado a alterações de padrões de metilação que podem estar subsequentemente relacionadas ao surgimento de patologias associadas à idade, como a DP23. Em um estudo foi analisado o padrão de metilação do DNA em todo o genoma através de amostras do córtex frontal e leucócitos do sangue periférico de indivíduos saudáveis e de pacientes com DP. Este revelou em ambas amostras uma desregulação significativa na metilação das ilhas CpG no grupo de pacientes com DP. Foi observado um 
padrão distinto de metilação envolvendo muitos genes previamente associados à $\mathrm{DP}^{24}$.

Jowaed et al. ${ }^{25}$ observaram em diferentes tecidos cerebrais (putâmen, córtex e substância nigra) de pacientes com DP esporádica, uma diminuição significativa na metilação do íntron 1 do gene SNCA (gene que codifica a proteína alfa-sinucleína) em comparação com controles saudáveis. Estes sugeriram a hipótese de que o aumento na síntese de alfa-sinucleína pode ser devido ao aumento da expressão de SNCA, consequente da hipometilação do mesmo. Outro estudo não foi capaz de detectar diferenças de metilação nas ilhas CpG do promotor do SNCA no córtex cingulado anterior ou no putâmen de pacientes com DP. No entanto, na substância nigra destes pacientes a metilação foi significativamente diminuída quando comparada aos controles $^{26}$. O padrão de metilação do íntron 1 do gene SNCA foi posteriormente investigado em amostras de sangue ${ }^{27}$, em células mononucleares do sangue periférico ${ }^{28}$ e em leucócitos de pacientes com $\mathrm{DP}^{29}$. Estes estudos relataram uma diminuição significativa na metilação do promotor do gene SNCA quando comparados aos indivíduos controles, corroborando assim, com os achados nos tecidos cerebrais dos estudos anteriores.

A DNA metiltransferase 1 (DNMT1) é a metiltransferase de manutenção, que preserva os padrões de metilação estabelecidos no início do desenvolvimento. É expressa no cérebro, localizada em sua maior parte no compartimento nuclear e constitui um alvo candidato para 
estudar os mecanismos de metilação do DNA associados ao envelhecimento ${ }^{30}$. Em um estudo onde investigou-se os mecanismos subjacentes à alteração da metilação do DNA na DP e na demência com corpos Lewy (DCL) foi constatado a redução dos níveis nucleares desta enzima no córtex frontal de pacientes com DP, de pacientes com DCL e em camundongos transgênicos. Além disso, a alfasinucleína foi identificada como possível sequestradora da DNMT1 do núcleo ao citoplasma. Esta alteração na localização subcelular da DNMT1 resultou então, na hipometilação global do DNA envolvendo ilhas CpG de SNCA e de outros genes ${ }^{31}$.

As modificações das histonas já foram descritas por desempenhar um papel importante no desenvolvimento, diferenciação e manutenção de neurônios dopaminérgicos ${ }^{32}$. No entanto, pouco se sabe sobre suas implicações na patogênese da DP. Em um estudo recente, neurônios dopaminérgicos isolados a partir do mesencéfalo de pacientes com DP revelaram níveis aumentados de acetilação das histonas em comparação com indivíduos controles, assim como em cultura de células humanas tratadas com 1-metil-4-fenilpiridínio (MPP+) e em tecidos cerebrais de camundongos tratados com 1-metil-4-fenil1,2,3,6-tetrahidropiridina (MPTP), um modelo experimental da DP. Além disso, os níveis das HDACs encontraram-se reduzidos tanto nas células cultivadas in vitro, nos modelos experimentais e nos tecidos cerebrais dos pacientes ${ }^{33}$. Song et al. ${ }^{34}$ examinaram 0 efeito do pesticida Dieldrin na 
acetilação de histonas em células neuronais dopaminérgicas. $\mathrm{O}$ pesticida induziu um aumento na acetilação das histonas H3 e H4. Camundongos foram expostos também ao Dieldrin, e constatou-se a hiperacetilação de histonas no corpo estriado e substância nigra destes animais. Um outro agente neurotóxico, o herbicida Paraquat, induziu o aumento da acetilação da histona H3 em células dopaminérgicas in vitro, no entanto, não alterou a acetilação da histona H4. Também foi mensurada neste estudo a atividade da HDAC, e esta apresentou uma redução significativa como resposta a exposição ao Paraquat ${ }^{35}$.

Os principais mecanismos epigenéticos envolvidos com a DP estão resumidos na Tabela 1 .

\section{CONCLUSÃO}

A DP, assim como as demais doenças neurodegenerativas, é um distúrbio complexo que ocorre através da interação entre fatores genéticos, ambientais, nutricionais e outros, juntamente com o processo de envelhecimento. Observamos nessa revisão que 0 desequilíbrio de marcadores de acetilação de histonas e metilação de DNA estão presentes em modelos experimentais e pacientes com DP. Uma vez que a epigenética pode ser alterada em resposta a pelo menos um destes fatores, está se tornando cada vez mais aceito o seu envolvimento nestas patologias. 
Tabela 1. Principais mecanismos epigenéticos envolvidos com a DP.

\begin{tabular}{|c|c|c|c|c|}
\hline Referência & $\begin{array}{l}\text { Mecanismo } \\
\text { Epigenético }\end{array}$ & Gene ou Enzima & Tipo de Amostra & Resultados \\
\hline [24] & $\begin{array}{l}\text { Metilação do } \\
\text { DNA }\end{array}$ & Todo o genoma & $\begin{array}{l}\text { Córtex frontal e } \\
\text { leucócitos de } \\
\text { sangue periférico de } \\
\text { pacientes com DP }\end{array}$ & $\begin{array}{l}\text { Desregulação na } \\
\text { metilação das ilhas } \\
\text { CpG }\end{array}$ \\
\hline$[25]$ & $\begin{array}{l}\text { Metilação do } \\
\text { íntron } 1\end{array}$ & Gene SNCA & $\begin{array}{l}\text { Putâmen, córtex e } \\
\text { SN de pacientes } \\
\text { com DP esporádica }\end{array}$ & $\begin{array}{l}\downarrow \text { na metilação do } \\
\text { íntron } 1 \text { do gene SNCA }\end{array}$ \\
\hline
\end{tabular}

\begin{tabular}{|c|c|c|c|c|}
\hline [26] & $\begin{array}{l}\text { Metilação do } \\
\text { íntron } 1\end{array}$ & Gene SNCA & $\begin{array}{lr}\text { Córtex } & \text { cingulado } \\
\text { anterior, putâmen e } \\
\text { SN de pacientes } \\
\text { com DP }\end{array}$ & $\begin{array}{l}\downarrow \text { da metilação nas ilhas } \\
\text { CpG do promotor do } \\
\text { SNCA na SN }\end{array}$ \\
\hline [27] & $\begin{array}{l}\text { Metilação } \\
\text { íntron } 1\end{array}$ & Gene SNCA & $\begin{array}{l}\text { Amostras de sangue } \\
\text { de pacientes com } \\
\text { DP }\end{array}$ & $\begin{array}{l}\text { \na metilação } \\
\text { promotor do } \\
\text { SNCA }\end{array}$ \\
\hline [28] & $\begin{array}{l}\text { Metilação } \\
\text { íntron } 1\end{array}$ & Gene SNCA & $\begin{array}{l}\text { Células } \\
\text { mononucleares de } \\
\text { sangue periférico de } \\
\text { pacientes com DP }\end{array}$ & $\begin{array}{l}\downarrow \text { na metilação do } \\
\text { promotor do gene SNCA }\end{array}$ \\
\hline [29] & $\begin{array}{l}\text { Metilação } \\
\text { íntron } 1\end{array}$ & Gene SNCA & $\begin{array}{l}\text { Leucócitos de } \\
\text { pacientes com DP }\end{array}$ & $\begin{array}{l}\downarrow \text { na metilação } \\
\text { promotor do } \\
\text { SNCA }\end{array}$ \\
\hline [31] & $\begin{array}{l}\text { Metilação do } \\
\text { DNA }\end{array}$ & Enzima DNMT1 & $\begin{array}{l}\text { Córtex frontal } \\
\text { pacientes com } \\
\text { com DCL e de } \\
\text { camundongos }\end{array}$ & $\begin{array}{l}\downarrow \text { dos níveis nucleares } \\
\text { da DNMT1, } \\
\text { hipometilação global do } \\
\text { DNA }\end{array}$ \\
\hline [33] & $\begin{array}{l}\text { Acetilação das } \\
\text { histonas }\end{array}$ & $\begin{array}{l}\text { Níveis de acetilação } \\
\text { das histonas H2A, } \\
\text { H3 e H4 e níveis } \\
\text { das HDACs }\end{array}$ & $\begin{array}{l}\text { Cultura de células } \\
\text { humanas, tecidos } \\
\text { cerebrais de } \\
\text { camundongos } \\
\text { neurônios } \\
\text { dopaminérgicos de } \\
\text { pacientes com DP }\end{array}$ & $\begin{array}{l}\text { } \uparrow \text { de acetilação das } \\
\text { histonas em todas as } \\
\text { amostras e níveis das } \\
\text { HDACs reduzidas }\end{array}$ \\
\hline [34] & $\begin{array}{l}\text { Acetilação das } \\
\text { histonas }\end{array}$ & $\begin{array}{l}\text { Níveis de acetilação } \\
\text { das histonas H3 e } \\
\text { H4 }\end{array}$ & $\begin{array}{l}\text { Cultura de células } \\
\text { neuronais } \\
\text { dopaminérgicas, } \\
\text { corpo estriado e SN } \\
\text { de camundongos } \\
\text { expostos ao Dieldrin }\end{array}$ & $\begin{array}{l}\uparrow \text { de acetilação das } \\
\text { histonas em todas as } \\
\text { amostras }\end{array}$ \\
\hline [35] & $\begin{array}{l}\text { Acetilação das } \\
\text { histonas }\end{array}$ & $\begin{array}{l}\text { Níveis de acetilação } \\
\text { das histonas H3 e } \\
\text { H4 e atividade da } \\
\text { HDAC }\end{array}$ & $\begin{array}{l}\text { Cultura de células } \\
\text { neuronais } \\
\text { dopaminérgicas } \\
\text { tratadas com } \\
\text { Paraquat }\end{array}$ & $\begin{array}{l}\uparrow \text { da acetilação da } \\
\text { histona H3 e } \downarrow \text { da } \\
\text { HDAC }\end{array}$ \\
\hline
\end{tabular}

DP: (Doença de Parkinson), DNMT1: (DNAmetiltransferase 1), DCL: (demência com corpos de Lewy), SN: (substância nigra), HDAC: (histona desacetilase), $\downarrow$ : diminuição, $\uparrow$ : aumento. 
Deste modo, esperamos que este campo forneça importantes mecanismos moleculares que possam contribuir para a compreensão da patogênese da DP. Maiores estudos que possibilitem 0 avanço nesse conhecimento são essenciais para o estabelecimento de biomarcadores para um diagnóstico prematuro e abordagens terapêuticas mais efetivas para esta patologia.

\section{AGRADECIMENTOS}

Os autores gostariam de agradecer pelas bolsas de pesquisa ao Conselho Nacional de Desenvolvimento Científico e Tecnológico (CNPq) e a Coordenação de Aperfeiçoamento de Pessoal Superior (CAPES).

\section{REFERÊNCIAS}

1.Thomas B. Molecular insights into Parkinson's disease. F1000 Med Rep 2011;3:7. https://dx.doi.org/10.3410\%2FM3-7

2.Wu Y, Le W, Jankovic J. Preclinical Biomarkers of Parkinson Disease. Arch Neurol 2011;68:22-30.

https://dx.doi.org/10.1001/archneurol.2010.321

3.Delenclos $M$, Jones DR, McLean PJ, Uitti RJ. Biomarkers in Parkinson's disease: Advances and strategies. Parkinsonism Relat Disord. 2016;22:106-10.

https://doi.org/10.1016/j.parkreldis.2015.09.048

4.Dexter $D$, Jenner $P$. Parkinson disease: from pathology to molecular disease mechanisms. Free Radic Biol Med 2013;62:132-44. https://doi.org/10.1016/j.freeradbiomed.2013.01.018

5.Feng Y, Liu T, Li X, Liu Y, Zhu X, Jankovic, et al. Neuroprotection by Orexin-A via HIF-1a induction in a cellular model of Parkinson's disease. Neurosci Lett 2014;579:35-40.

https://doi.org/10.1016/j.neulet.2014.07.014

6.Desplats $P$, Patel P, Kosberg K, Mante M, Patrick C, Rockenstein E, et al. Combined exposure to Maneb and Paraquat alters transcriptional regulation of neurogenesis-related genes in mice 
models of Parkinson's disease. Mol Neurodegener 2012;7:49. https://doi.org/10.1186/1750-1326-7-49

7.Saha RN, Pahan K. HATs and HDACs in neurodegeneration: a tale of disconcerted acetylation homeostasis. Cell Death Differ 2006;13:539-50. https://doi.org/10.1038/sj.cdd.4401769

8. Elsner V, Basso C, Bertoldi K, de Meireles L, Cechinel L, Siqueira I. Differential effect of treadmill exercise on histone deacetylase activity in rat striatum at different stages of development. J of Physiol Sci 2017;67:387-94. https://doi.org/10.1007/s12576-016-0471-2

9.Lavratti C, Dorneles G, Pochmann D, Peres A, Bard A, de Lima Schipper $L$, et al. Exercise-induced modulation of histone H4 acetylation status and cytokines levels in patients with schizophrenia. Physiol Behav. 2017;168:84-90.

https://doi.org/10.1016/j.physbeh.2016.10.021

10.Portela A, Esteller M. Epigenetic modifications and human disease. Nat Biotechnol 2010;28:1057-68. https://doi.org/10.1038/nbt.1685 11.Schumacher A. Aging Epigenetics. In: Tollefsbol, T. (ed.). Handbook of Epigenetics. $2^{\text {nd }}$ ed. San Francisco: Elsevier, 2011, p.405-19.

12. AmmalKaidery N, Tarannum S, Thomas B. Epigenetic Landscape of Parkinson's Disease: Emerging Role in Disease Mechanisms and Therapeutic Modalities. Neurotherapeutics 2013;10:698-708. https://doi.org/10.1007/s13311-013-0211-8

13. Day JJ, Sweatt JD. DNA methylation and memory formation. Nat Neurosci 2010;13:1319. https://doi.org/10.1038/nn.2666

14.Guo J, Ma D, Mo H, Ball M, Jang M, Bonaguidi M, et al. Neuronal activity modifies the DNA methylation landscape in the adult brain. Nat Neurosci 2011;14:1345-51. https://doi.org/10.1038/nn.2900 15. Mikeska T, Craig J. DNA Methylation Biomarkers: Cancer and Beyond. Genes 2014;5:821-64.

https://dx.doi.org/10.3390\%2Fgenes5030821

16.Wagner W, Fernandez-Rebollo E, Frobel J. DNA-methylation changes in replicative senescence and aging: two sides of the same coin? Epigenomics 2016;8:1-3. https://doi.org/10.2217/epi.15.100

17. Labbé C, Lorenzo-Betancor 0 , Ross 0 . Epigenetic regulation in Parkinson's disease. Acta Neuropathol 2016;132:515-30.

https://dx.doi.org/10.1007\%2Fs00401-016-1590-9

18.Dell'Aversana C, Lepore I, Altucci L. HDAC modulation and cell death in the clinic. Exp Cell Res 2012;318:1229-44. https://doi.org/10.1016/j.yexcr.2012.01.025

19.Sadakierska-Chudy A, Filip M. A Comprehensive View of the Epigenetic Landscape. Part II: Histone Post-translational Modification, Nucleosome Level, and Chromatin Regulation by ncRNAs. Neurotox Res 2014;27:172-97. https://doi.org/10.1007/s12640-014-9508-6 20.Blanch M, Mosquera JL, Ansoleaga B, Ferrer I, Barrachina M. Altered mitochondrial DNA methylation pattern in Alzheimer diseaserelated pathology and in Parkinson disease. Am J Pathol 2016;186:385-97. https://doi.org/10.1016/j.ajpath.2015.10.004 
21. Horvath S. DNA methylation age of human tissues and cell types. Genome Biol 2013;14:R115. https://doi.org/10.1186/gb-2013-14$10-r 115$

22.Qureshi I, Mehler M. Advances in Epigenetics and Epigenomics for Neurodegenerative Diseases. Curr Neurol Neurosci Rep 2011;11:46473. https://doi.org/10.1007/s11910-011-0210-2

23.Poulopoulos M, Levy O, Alcalay R. The neuropathology of genetic Parkinson's disease. Mov Disord 2012;27:831-42. https://doi.org/10.1002/mds.24962

24. Masliah E, Dumaop W, Galasko D, Desplats P. Distinctive patterns of DNA methylation associated with Parkinson disease. Epigenetics 2013;8:1030-8. https://doi.org/10.4161/epi.25865

25.Jowaed A, Schmitt I, Kaut O, Wullner U. Methylation Regulates Alpha-Synuclein Expression and Is Decreased in Parkinson's Disease Patients' Brains. J Neurosci 2010;30:6355-59.

https://doi.org/10.1523/JNEUROSCI.6119-09.2010

26. Matsumoto L, Takuma $H$, Tamaoka A, Kurisaki $H$, Date $H$, Tsuji S, et al. CpG Demethylation Enhances Alpha-Synuclein Expression and Affects the Pathogenesis of Parkinson's Disease. PLoS ONE 2010;5:e15522. https://doi.org/10.1371/journal.pone.0015522

27.Pihlstrøm L, Berge V, Rengmark A, Toft M. Parkinson's disease correlates with promoter methylation in the a-synuclein gene. Mov Disord 2014;30:577-80. https://doi.org/10.1002/mds.26073

28. Ai SX, Xu Q, Hu YC, Song CY, Guo JF, Shen L, et al. Hypomethylation of SNCA in blood of patients with sporadic Parkinson's disease. J Neurol Sci 2014;337:123-8. https://doi.org/10.1016/j.jns.2013.11.033

29.Tan Y, Wu L, Zhao Z, Wang Y, Xiao Q, Liu J, et al. Methylation of a-synuclein and leucine-rich repeat kinase 2 in leukocyte DNA of Parkinson's disease patients. Parkinsonism Relat Disord. 2014;20:308-13. https://doi.org/10.1016/j.parkreldis.2013.12.002 30.Singh V, Sharma P, Capalash N. DNA Methyltransferase-1 Inhibitors as Epigenetic Therapy for Cancer. Curr Cancer Drug Tar 2013;13:379-99. http://dx.doi.org/10.2174/15680096113139990077 31.Desplats P, Spencer B, Coffee E, Patel P, Michael S, Patrick C, et al. a-Synuclein Sequesters Dnmt1 from the Nucleus. J Biol Chem 2011;286:9031-7. https://doi.org/10.1074/jbc.C110.212589

32. vanHeesbeen $\mathrm{H}$, Mesman S, Veenvliet J, Smidt M. Epigenetic mechanisms in the development and maintenance of dopaminergic neurons. Development 2013;140:1159-69.

https://doi.org/10.1242/dev.089359

33.Park G, Tan J, Garcia G, Kang Y, Salvesen G, Zhang Z. Regulation of Histone Acetylation by Autophagy in Parkinson Disease. J Biol Chem 2015;291:3531-40. https://doi.org/10.1074/jbc.M115.675488 34.Song C, Kanthasamy A, Anantharam V, Sun F, Kanthasamy A. Environmental Neurotoxic Pesticide Increases Histone Acetylation to Promote Apoptosis in Dopaminergic Neuronal Cells: Relevance to Epigenetic Mechanisms of Neurodegeneration. Mol Pharmacol 2010;77:621-32. https://doi.org/10.1124/mol.109.062174 
35.Song C, Kanthasamy A, Jin $H$, Anantharam V, Kanthasamy A. Paraquat induces epigenetic changes by promoting histone acetylation in cell culture models of dopaminergic degeneration. NeuroToxicology 2011;32:586-95.

https://doi.org/10.1016/j.neuro.2011.05.018 